\title{
Low methylation levels of the $S F R P 1$ gene are associated with the basal-like subtype of breast cancer
}

\author{
YOUNG JU JEONG ${ }^{1}$, HYE YEON JEONG $^{1}$, JIN GU BONG $^{1}$, SUNG HWAN PARK $^{1}$ and HOON KYU OH ${ }^{2}$ \\ Departments of ${ }^{1}$ Surgery and ${ }^{2}$ Pathology, College of Medicine, \\ Catholic University of Daegu, Daegu 705-718, Republic of Korea
}

Received December 17, 2012; Accepted February 15, 2013

DOI: $10.3892 /$ or.2013.2335

\begin{abstract}
Epigenetic analyses have shown that aberrant DNA methylation signatures are associated with breast cancer molecular subtypes. In this study, we analyzed the methylation status of breast cancer-related genes in relation to the molecular subtypes and investigated whether the basal-like subtype displays distinct methylation profiles. By using pyrosequencing, we analyzed the DNA methylation status of 5 candidate genes in 60 breast cancer samples. We compared the methylation frequency across the molecular subtypes and analyzed the correlation between methylation levels and clinicopathological characteristics. A total of 59 cases displayed aberrant methylation. Amplification during polymerase chain reaction analysis failed in 1 case. The median methylation levels of the secreted frizzled-related protein 1 (SFRPI) gene were significantly lower in the basal-like subtype compared to the luminal A, luminal B and human epidermal growth factor receptor 2 (HER2) subtypes. Cadherin 13 (H-cadherin; CDH13) methylation levels were significantly higher in the HER2 tumors compared to the luminal A and basal-like subtypes. A comparison of the methylation status with clinicopathological characteristics revealed that the expression of $\mathrm{Bcl}-2$, progesterone receptor and epidermal growth factor receptor were associated with SFRPI gene methylation status. Our results indicate that the basal-like subtype is associated with low methylation levels of the SFRPI gene, suggesting that the methylation levels of specific breast cancer genes may potentially serve as epigenetic biomarkers and prognostic factors.
\end{abstract}

\section{Introduction}

Breast cancer is a molecularly, biologically and clinically heterogeneous disease. Previous microarray profiling studies

Correspondence to: Professor Young Ju Jeong, Department of Surgery, College of Medicine, Catholic University of Daegu, 3056-6 Daemyoung-4-dong, Nam-gu, Daegu 705-718, Republic of Korea E-mail: yjjeong@cu.ac.kr

Key words: breast cancer, basal-like, methylation, secreted frizzledrelated protein 1 , epigenetics on breast cancer have identified subtypes that are associated with different clinical outcomes $(1,2)$. These subtypes are classified as luminal A, luminal B, human epidermal growth factor receptor 2 (HER2), basal-like and normal-like subtypes. The identification of gene expression-based breast cancer subtypes is considered a critical means of prognostication (3) and the basal-like subtype is an aggressive tumor that has a poor prognosis and no specific targeted therapy. Since it is not always feasible to obtain gene expression array information, a simple classification using a combination of immunohistochemical markers has been proposed and adopted in clinical practice $(4,5)$. However, there is still a need for well-defined biomarker panels that allow breast cancer subtyping for clinical diagnostics and the management of the disease.

Breast carcinogenesis is a multistep process resulting from the accumulation of genetic alterations, as well as epigenetic changes, such as promoter methylation and histone modification $(6,7)$. Promoter hypermethylation at specific gene loci leading to gene silencing is a major mechanism of epigenetic inactivation in cancer cells. A previous genome-wide study on breast cancer has led to the identification of a number of tumor suppressor genes that are inactivated by promoter hypermethylation (8), and epigenetic analyses have shown aberrant DNA methylation signatures associated with the molecular subtypes of breast cancer $(9,10)$. However, limited information is available on the methylation status of candidate genes associated with each molecular subtype.

The purpose of this study was to analyze the methylation status of breast cancer-related genes according to the molecular subtypes found in Korean women, and to investigate whether the basal-like subtype displays distinct methylation profiles compared with the other subtypes. We included 5 genes that are involved in breast carcinogenesis and are commonly methylated in breast cancer [cadherin 13 (H-cadherin; $C D H 13)$, secreted frizzled-related protein 1 (SFRPI), fragile histidine triad (FHIT), Syk and retinoblastoma protein-interacting zinc-finger gene $1(R I Z 1)]$ and analyzed the methylation status of these genes using a sensitive and quantitative pyrosequencing assay.

\section{Materials and methods}

Patients and tumor characteristics. A total of 60 sporadic invasive ductal carcinoma (IDC) tissue samples were obtained from the Daegu Catholic University Hospital (Daegu, Korea). 
Each sample represented the 4 major molecular subtypes, encompassing the basal-like, HER2, luminal A and luminal B subtypes. All specimens were reviewed by an experienced pathologist. We subclassified the breast cancer samples according to immunohistochemical findings for the estrogen receptor (ER), progesterone receptor (PR), HER2 oncogene and Ki-67 labeling index (11). The basal-like subtype was defined as HER2-negative, ER- and PR-negative (triple-negative) breast cancer. The HER2 subtype was defined as HER2-positive, ER- and PR-negative. The luminal B subtype was defined as HER2-positive and ER- and/or PR-positive breast cancer (HER2-positive). The luminal A subtype was defined as ERand/or PR-positive, HER2-negative breast cancer with a low Ki-67 index. ER- and/or PR-positive, HER2-negative breast cancer with a high $\mathrm{Ki}-67$ status was classified as the luminal B (HER2-negative) subtype. Ethics approval for the study was obtained from the institutional review board at Daegu Catholic University Hospital.

Construction of tissue microarrays (TMAs). Representative paraffin tumor blocks were selected according to the primary evaluation of hematoxylin and eosin (H\&E)-stained slides before they were prepared for TMA analysis. Two tumor tissue cores $(1 \mathrm{~mm}$ in diameter) were taken from each of the donor breast cancer tissue blocks using a manual punch arrayer (Quick-Ray ${ }^{\mathrm{TM}}$; Uni-Tech Science, Seoul, Korea). The cores were placed in a new recipient paraffin block that ultimately contained 72-96 tissue cores. Each array block contained both tumor and control tissue samples. Multiple sections (5- $\mu \mathrm{m}$-thick) were cut from the TMA blocks and then mounted onto microscope slides. The TMA H\&E-stained sections were reviewed under a light microscope to confirm the presence of representative tumor areas.

Immuohistochemical staining and interpretation. Immunohistochemical analysis was performed on 5- $\mu$ m-thick TMA tissue sections using the Bond Polymer Intense Detection system (Leica Microsystems, Mount Waverley, Victoria, Australia) according to the manufacturer's instructions with minor modifications. Briefly, the 5- $\mu \mathrm{m}$-thick sections of formalin-fixed and paraffin-embedded TMA tissues were deparaffinized with Bond Dewax Solution (Leica Microsystems), and an antigen retrieval procedure was performed using Bond ER Solution (Leica Microsystems) for $30 \mathrm{~min}$ at $100^{\circ} \mathrm{C}$. The endogenous peroxidase was quenched by a 5-min incubation with hydrogen peroxide. Sections were incubated for $15 \mathrm{~min}$ at an ambient temperature with commercially available primary monoclonal antibodies for ER (1:100, clone 6F11; Novocastra), PR (1:100, clone 16; Novocastra), HER2 (1:250, A0485; Dako), Ki-67 (1:200, MM1-L; Novocastra), Bcl-2 (1:4, clone 124; Dako), p53 (1:200, BP53.12; Zymed) and epidermal growth factor receptor (EGFR) (1:100, clone EGFR.25; Novocastra) using a biotin-free polymeric horseradish peroxidase-linker antibody conjugate system in a Bond-Max automatic slide stainer (Leica Microsystems).

A cut-off value of $10 \%$ for the stained nuclei was used to define ER and PR positivity. Cytoplasmic staining of any intensity in $>10 \%$ of the tumor cells was scored as positive for Bcl-2. Membranous staining for HER-2 with strong complete staining in $10 \%$ of the tumor cells was regarded as HER-2 overexpression. p53 staining was scored positive if $>10 \%$ of the cells were stained with a strong intensity. The Ki-67 labeling index was expressed as a percentage and was graded as 'high' if the number of positive cells was $\geq 14 \%$.

DNA extraction and sodium bisulfate treatment. For DNA extraction, 8 tissue sections (5-10- $\mu$ m-thick) were obtained from the paraffin-embedded primary breast cancer tissues. Genomic DNA was isolated using the QIAamp DNA FFPE Tissue kit (Qiagen, Hilden, Germany) following the manufacturer's instructions. The purified DNA was quantified using a ND-1000 spectrophotometer (NanoDrop Technologies, Inc., Wilmington, DE, USA). The quality of the DNA was verified by gel electrophoresis. Sodium bisulfate modification of 200-500 ng genomic DNA was performed using the EZ DNA Methylation-Gold kit (Zymo Research, Orange, CA, USA) according to the manufacturer's instructions.

Candidate selection. Over 100 individual candidate genes have been reported to be commonly hypo- and hypermethylated in breast cancer. We carried out literature searches on PubMed http://www.ncbi.nlm.nih.gov/pubmed for the keywords (breast cancer, cancer and methylation) and searched serial analysis of gene expression (SAGE) data (GeneCards, http://www. genecards.org/cgi-bin/cardsearch). We selected 5 genes, cadherin 13 (H-cadherin; $C D H 13$ ), secreted frizzled-related protein 1 (SFRPI), FHIT, Syk and RIZ1, and functional annotation of the candidate genes was carried out using the functional annotation table function in the DAVID database http://david. abcc.ncifcrf.gov (12).

Pyrosequencing. Methylation analysis was carried out using pyrosequencing. Primers were designed using the PyroMark Assay Design program version 2.0.1.15 (Qiagen) and the sequences are presented in Table I. Polymerase chain reaction (PCR) was carried out using bisulfate-treated DNA under the following conditions: $95^{\circ} \mathrm{C}$ for $5 \mathrm{~min} ; 45$ cycles of $95^{\circ} \mathrm{C}$ for $30 \mathrm{sec}, 55^{\circ} \mathrm{C}$ for $30 \mathrm{sec}$ and $72^{\circ} \mathrm{C}$ for $30 \mathrm{sec}$; and a final extension of $5 \mathrm{~min}$ at $72^{\circ} \mathrm{C}$. PCR was conducted using a PCR premix (Enzynomics, Daejeon, Korea), and the quality and quantity of the PCR product was confirmed by performing agarose gel (2\%) electrophoresis with loading $4 \mu \mathrm{l}$ of 20 PCR products. Pyrosequencing was performed using the Pyro Gold kit and PSQ 96MA instrument (Qiagen) as instructed by the manufacturer. The methylation index (MtI) of each gene in each sample was calculated as the average value of ${ }^{\mathrm{m}} C /\left({ }^{\mathrm{m}} C+C\right)$ for all examined $\mathrm{CpG}$ sites in target regions. All experiments included a negative control without a template.

Statistical analysis. Statistical analyses were carried out using SPSS version 15.0 software (SPSS Inc., Chicago, IL, USA). A one-sample Kolmogorov-Smirnov test was used to evaluate the normal distribution fit of continuous parameters. The clinicopathological characteristics were compared across the 4 different breast cancer subtypes using the $\chi^{2}$ test or Fisher's exact test for categorical data, and ANOVA or the non-parametric Kruskal-Wallis test for continuous data. A comparison of the mean methylation frequencies across the subtypes was performed using ANOVA or the KruskalWallis test, and distributions of methylation levels across the 
Table I. Primer sequences used for PCR and pyrosequencing.

\begin{tabular}{ll}
\hline Primername & \multicolumn{1}{c}{ Primer sequence $\left(5^{\prime} \rightarrow 3^{\prime}\right)$} \\
\hline CDH13_F & TAAGGAAAATATGTTTAGTGTAGT \\
CDH13_R & AAATTCTCCACTACATTTTATCC \\
CDH13_S & GTGTAGTAGAGTGTATGAATGAAAA \\
SFRP1_F & TTTTAGGAGGTTTTTGGAAGT \\
SFRP1_R & ACTCTACCCCCTATTCTCC \\
SFRP1_S & AGGTTTTTGGAAGTTTG \\
FHIT_F & GGGAGGTAAGTTTAAGTGGAATATTG \\
FHIT_R & CCACTAAACTCCCAAATAATAACCTAAC \\
FHIT_S & GTAAGTTTAAGTGGAATATTGT \\
Syk_F & TTAGTAGGGAGGGTTAGGG \\
Syk_R & CTCATTTTAAACAACTTCCTTAAC \\
Syk_S & ATATTGGGAGGAAGTG \\
RIZ1_F & AGTAAGTTTTTTAAGGGTAGGATTAT \\
RIZ1_R & CCCTAATACCCAAAAACAATAACCAA \\
RIZ1_S & GTTTTTTAAGGGTAGGATTATTAT \\
\hline
\end{tabular}

CDH13, cadherin 13 (H-cadherin); SFRP1, secreted frizzled-related protein 1; RIZ1, retinoblastoma protein-interacting zinc-finger gene 1 . F, forward primer for PCR, R, reverse primer for PCR; S, primer used for pyrosequencing.

different subtypes were depicted for each gene using box plots. Associations between methylation status and clinicopathological characteristics were assessed using the Student's t-test or the non-parametric Mann-Whitney $U$ test for categorical variables, and correlation between 2 continuous variables was assessed using correlation analysis. All tests were two-sided and a P-value $<0.05$ was considered to indicate a statistically significant difference.

Clustering analyses were performed using GeneSpring GX version 7.3 (Agilent Technologies Inc., Santa Clara, CA, USA) on the basis of the mean methylation levels of genes and the $\mathrm{CpG}$ sites. Hierarchical clustering was performed using Pearson's correlation distance and average linkage.

\section{Results}

Clinicopathological characteristics. The patient characteristics are presented in Table II. The average age of the 60 patients with invasive breast cancer was $51.77 \pm 13.22$ years (range, 26-90 years). A total of 15 cases were included for each molecular subtype in the 60 breast cancer samples. TNM staging was as follows: stage I, 29 patients (48.3\%); stage II, 21 patients $(35.0 \%)$; stage III, 6 patients $(10.0 \%)$; and stage IV, 4 patients $(6.7 \%)$.

Table III presents the clinicopathological characteristics according to the 4 breast cancer subtypes. The basal subtype was characterized by a high histologicical grade $(\mathrm{P}<0.001)$, low extensive intraductal component (EIC) $(\mathrm{P}<0.001)$, the presence of necrosis $(\mathrm{P}<0.001)$, a high Ki-67 level $(\mathrm{P}<0.001)$ and a positive expression of EGFR $(\mathrm{P}<0.001)$.
Table II. General patient characteristics

Characteristics Value

Age (years), mean (range)

$51.77 \pm 13.22(26-90)$

Menopausal status, n (\%)

Pre-menopausal

Post-menopausal

Tumor size $(\mathrm{cm})$, mean (range)

$1.80 \pm 0.93(0.10-4.50)$

Histological grade, n (\%)

I

$13(21.7)$

$11(18.3)$

III

$36(60.0)$

Nodal involvement, n (\%)

Negative

$40(69.0)$

Positive

$18(31.0)$

Distant metastasis, n (\%)

Negative

58 (96.7)

Positive

$15(25.0)$

$15(25.0)$

$15(25.0)$

$15(25.0)$

Basal-like

Methylation level of candidate gene, mean $\%$

CDH13
SFRP1
FHIT
Syk
RIZ1

$15.66 \pm 13.84$

$15.67 \pm 11.21$

$3.43 \pm 0.97$

$8.73 \pm 5.32$

$48.30 \pm 11.55$

Lymphovascular invasion, n (\%)

Negative

$39(66.1)$

Positive

$20(33.9)$

ER, n (\%)

Negative

$31(51.7)$

Positive

29 (48.3)

PR, n (\%)

Negative

$33(55.0)$

Positive

27 (45.0)

HER2 overexpression, n (\%)

Negative

$30(50.0)$

Positive

$30(50.0)$

$\mathrm{Ki}-67, \mathrm{n}(\%)$

$<14 \%$

$25(41.7)$

$\geq 14 \%$

35 (58.3)

HER2, human epidermal growth factor receptor 2; $C D H 13$, cadherin 13 (H-cadherin); SFRP1, secreted frizzled-related protein 1; RIZ1, retinoblastoma protein-interacting zinc-finger gene 1; ER, estrogen receptor PR, progesterone receptor. 
Table III. Clinicopathological characteristics according to breast cancer subtype.

\begin{tabular}{|c|c|c|c|c|c|}
\hline \multirow[b]{2}{*}{ Characteristics } & \multicolumn{5}{|c|}{ Subtype } \\
\hline & Luminal A & Luminal B & HER2 & Basal-like & P-value \\
\hline Age (years), mean \pm SD & $57.3 \pm 15.2$ & $46.9 \pm 7.1$ & $50.3 \pm 11.8$ & $52.6 \pm 15.9$ & 0.273 \\
\hline \multicolumn{6}{|l|}{ Menopausal status, n (\%) } \\
\hline Pre-menopausal & 7 (46.7) & $8(57.1)$ & $7(46.7)$ & $5 \quad(33.3)$ & \multirow[t]{2}{*}{0.643} \\
\hline Post-menopausal & $8 \quad(53.3)$ & $6(42.9)$ & $8(53.3)$ & $10(66.7)$ & \\
\hline Tumor size $(\mathrm{cm})$, mean \pm SD & $1.6 \pm 0.7$ & $1.6 \pm 1.0$ & $1.7 \pm 0.8$ & $2.3 \pm 1.0$ & 0.082 \\
\hline \multicolumn{6}{|l|}{ Histological grade, n (\%) } \\
\hline I & $9(60.0)$ & $2(13.3)$ & $2(15.4)$ & $0 \quad(0.0)$ & \multirow[t]{3}{*}{$<0.001$} \\
\hline II & $5 \quad(33.3)$ & $4 \quad(26.7)$ & $2(13.3)$ & $0 \quad(0.0)$ & \\
\hline III & $1 \quad(6.7)$ & $9(60.0)$ & $11(73.3)$ & $15(100.0)$ & \\
\hline \multicolumn{6}{|l|}{ Nodal involvement, $\mathrm{n}(\%)$} \\
\hline Negative & $10(66.7)$ & $10(66.7)$ & $12(85.7)$ & $8 \quad(57.1)$ & \multirow[t]{2}{*}{0.432} \\
\hline Positive & $5(33.3)$ & $5(33.3)$ & $2(14.3)$ & $6(42.9)$ & \\
\hline \multicolumn{6}{|l|}{ Distant metastasis, n (\%) } \\
\hline Negative & $15(100.0)$ & $15(100.0)$ & $14(93.3)$ & $14(93.3)$ & \multirow[t]{2}{*}{1.000} \\
\hline Positive & $\begin{array}{ll}0 & (0.0)\end{array}$ & $0 \quad(0.0)$ & 1 (6.7) & $1 \quad(6.7)$ & \\
\hline \multicolumn{6}{|l|}{ Lymphovascular invasion, $\mathrm{n}(\%)$} \\
\hline Negative & $10(66.7)$ & $10(66.7)$ & $11(73.3)$ & $8(57.1)$ & \multirow[t]{2}{*}{0.844} \\
\hline Positive & $5(33.3)$ & $5(33.3)$ & $4 \quad(26.7)$ & $6 \quad(42.9)$ & \\
\hline $\mathrm{EIC}(\%)$, mean $\pm \mathrm{SD}$ & $13.9 \pm 19.7$ & $31.7 \pm 38.8$ & $12.9 \pm 37.7$ & $3.6 \pm 9.5$ & $<0.001$ \\
\hline \multicolumn{6}{|l|}{ Necrosis, $\mathrm{n}(\%)$} \\
\hline Negative & $14(100.0)$ & $11(73.3)$ & $6(40.0)$ & $1 \quad(12.5)$ & \multirow[t]{2}{*}{$<0.001$} \\
\hline Positive & $0 \quad(0.0)$ & $4 \quad(26.7)$ & $9(60.0)$ & $7 \quad(87.5)$ & \\
\hline \multicolumn{6}{|l|}{$\mathrm{Ki}-67, \mathrm{n}(\%)$} \\
\hline$<14 \%$ & $15(100.0)$ & $6 \quad(40.0)$ & $4 \quad(26.7)$ & $0 \quad(0.0)$ & \multirow[t]{2}{*}{$<0.001$} \\
\hline$\geq 14 \%$ & $0 \quad(0.0)$ & $9(60.0)$ & $11 \quad(73.3)$ & $15(100.0)$ & \\
\hline \multicolumn{6}{|l|}{$\mathrm{ER}, \mathrm{n}(\%)$} \\
\hline Negative & $0 \quad(0.0)$ & $1 \quad(6.7)$ & $15(100.0)$ & $15(100.0)$ & \multirow[t]{2}{*}{$<0.001$} \\
\hline Positive & $15(100.0)$ & $14(93.3)$ & $\begin{array}{ll}0 & (0.0)\end{array}$ & $0 \quad(0.0)$ & \\
\hline \multicolumn{6}{|l|}{$\mathrm{PR}, \mathrm{n}(\%)$} \\
\hline Negative & $1 \quad(6.7)$ & $2(13.3)$ & $15(100.0)$ & $15(100.0)$ & \multirow[t]{2}{*}{$<0.001$} \\
\hline Positive & $14(93.3)$ & $13(86.7)$ & $\begin{array}{ll}0 & (0.0)\end{array}$ & $0 \quad(0.0)$ & \\
\hline \multicolumn{6}{|l|}{ HER $2, \mathrm{n}(\%)$} \\
\hline Negative & $15(100.0)$ & $0 \quad(0.0)$ & $\begin{array}{ll}0 & (0.0)\end{array}$ & $15(100.0)$ & \multirow[t]{2}{*}{$<0.001$} \\
\hline Positive & $\begin{array}{ll}0 & (0.0)\end{array}$ & $15(100.0)$ & $15(100.0)$ & $\begin{array}{ll}0 & (0.0)\end{array}$ & \\
\hline \multicolumn{6}{|l|}{ Bcl-2, n (\%) } \\
\hline Negative & $13(86.7)$ & $15(27.3)$ & $13(86.7)$ & 14 (93.3) & \multirow[t]{2}{*}{0.740} \\
\hline Positive & $2(13.3)$ & $\begin{array}{ll}0 & (0.0)\end{array}$ & $2(13.3)$ & $1 \quad(6.7)$ & \\
\hline p53, n (\%) & & & & & \\
\hline Negative & $2(13.3)$ & $1 \quad(6.7)$ & $3(20.0)$ & $5 \quad(33.3)$ & 0.353 \\
\hline Positive & $13(86.7)$ & $14(93.3)$ & $12(80.0)$ & $10 \quad(66.7)$ & \\
\hline EGFR, n (\%) & & & & & \\
\hline Negative & $14(93.3)$ & $14(93.3)$ & $7(50.0)$ & $\begin{array}{ll}0 & (90.0)\end{array}$ & $<0.001$ \\
\hline Positive & $1 \quad(6.7)$ & $1 \quad(6.7)$ & $7(50.0)$ & $15(100.0)$ & \\
\hline
\end{tabular}

EIC, extensive intraductal component; ER, estrogen receptor; PR, progesterone receptor; HER2, HER2, human epidermal growth factor receptor 2; EGFR, epidermal growth factor receptor. 

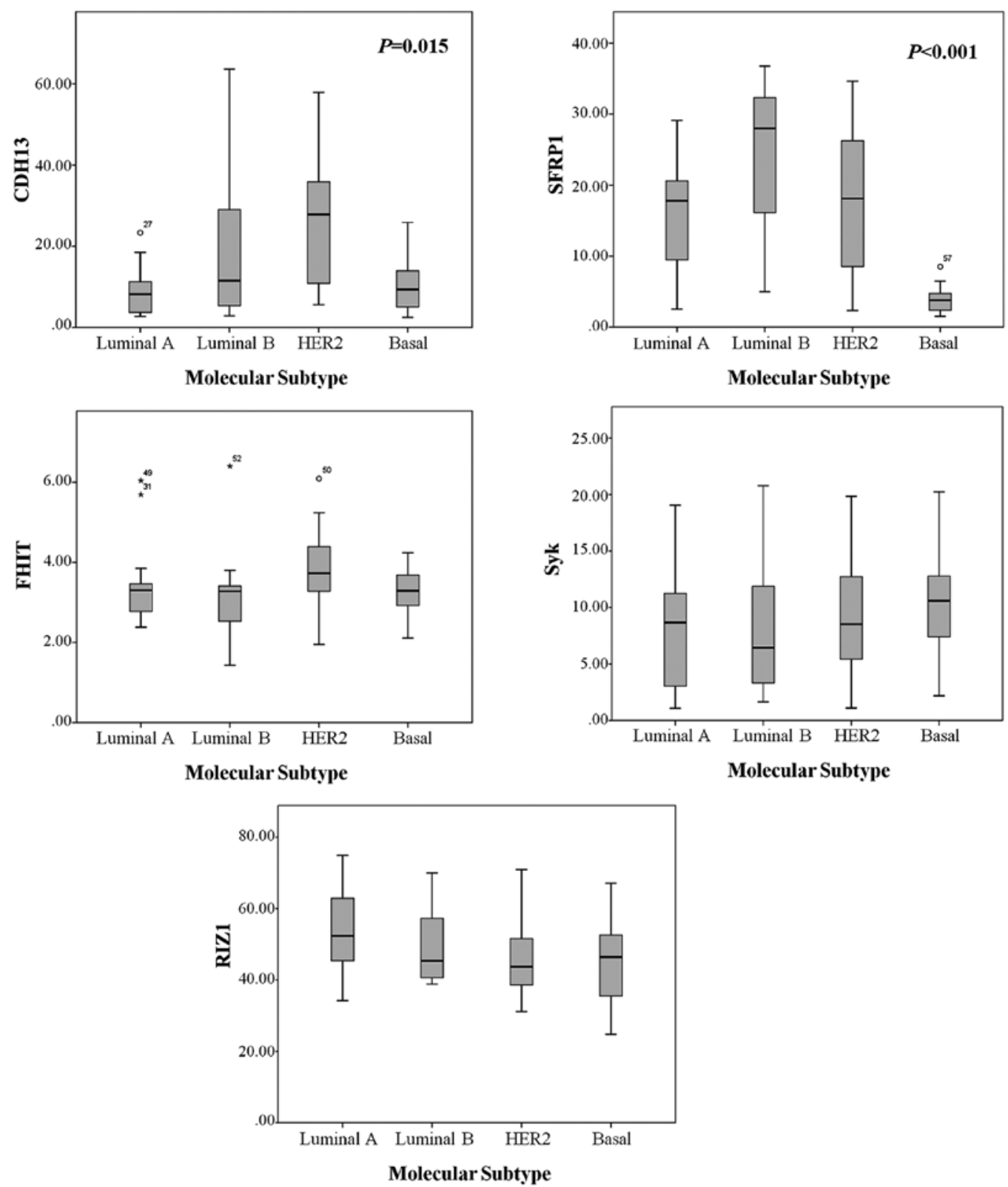

Figure 1. Distribution of gene methylation across breast cancer molecular subtypes. Box plots show distribution of individual gene methylation across the 4 breast cancer subtypes. The $C D H 13$ methylation level was significantly higher in HER2 tumors and the SFRP1 methylation level was significantly lower in the basal-like subtype compared to the other subtypes. $C D H 13$, cadherin 13 (H-cadherin); SFRP1, secreted frizzled-related protein 1; RIZ1, retinoblastoma protein-interacting zinc-finger gene 1 .

Methylation levels in different molecular subtypes of breast cancer. A total of 59 cases showed aberrantly methylated genes. Amplification of 1 sample failed during PCR analysis. The mean methylation levels of CDH13, SFRPI, FHIT, Syk and $R I Z 1$ were $15.66 \pm 13.84,15.67 \pm 11.21,3.43 \pm 0.97,8.73 \pm 5.32$ and $48.30 \% \pm 11.55 \%$, respectively. The methylation status of $C D H 13$ and $S F R P 1$ was significantly different according to breast cancer molecular subtypes (Table IV, Fig. 1). The $\mathrm{CDH} 13$ methylation level was significantly higher in HER2 tumors compared to the luminal and basal-like subtypes $(\mathrm{P}=0.006$ and $\mathrm{P}=0.012$, respectively) (Table V). The median methylation level and average methylation ratio of the SFRPI gene were significantly lower in the basal-like subtype compared to the luminal A, luminal B and HER2 subtypes $(\mathrm{P}=0.002, \mathrm{P}<0.001$ and $\mathrm{P}=0.003$, respectively).
FHIT, Syk and RIZ1 methylation levels showed no significant differences among the molecular subtypes.

Gene-specific patterns of methylation for classification of breast cancer. The varying methylation frequencies of the $C D H 13$ and SFRPI genes in the different breast cancer molecular subtypes provides evidence for subtype-specific methylation profiling in breast cancer classification. In particular, a low frequency of $S F R P 1$ methylation was significantly associated with the basal-like subtype $(\mathrm{P}<0.001)$. We determined the area under the curve (AUC) of the receiver operating characteristic (ROC) curve for the mean methylation level of the SFRP1 gene. For the cut-off value of $7.50 \%$, the AUC was 0.941 , with a sensitivity of $91.7 \%$ and a specificity of $84.2 \%$. 
Table IV. Methylation levels in breast cancer subtypes.

Methylation level (mean \pm SD)

\begin{tabular}{|c|c|c|c|c|c|}
\hline Gene & Luminal A & Luminal B & HER2 & Basal-like & P-value \\
\hline CDH13 & $9.2 \pm 5.9$ & $18.2 \pm 17.4$ & $25.1 \pm 16.2$ & $11.1 \pm 7.7 .8$ & 0.015 \\
\hline$S F R P 1$ & $15.5 \pm 7.8$ & $24.3 \pm 10.7$ & $18.0 \pm 10.7$ & $4.0 \pm 2.0$ & $<0.001$ \\
\hline FHIT & $3.4 \pm 1.1$ & $3.2 \pm 1.1$ & $3.8 \pm 1.0$ & $3.3 \pm 0.6$ & 0.367 \\
\hline Syk & $7.7 \pm 5.3$ & $8.2 \pm 5.7$ & $9.0 \pm 5.2$ & $10.4 \pm 5.2$ & 0.626 \\
\hline RIZ1 & $53.0 \pm 11.5$ & $48.7 \pm 10.9$ & $46.2 \pm 11.4$ & $44.5 \pm 12.1$ & 0.257 \\
\hline
\end{tabular}

HER2, human epidermal growth factor receptor 2; CDH13, cadherin 13 (H-cadherin); SFRP1, secreted frizzled-related protein 1; RIZ1, retinoblastoma protein-interacting zinc-finger gene 1 .

Table V. Comparison of methylation levels between breast cancer subtypes.

\begin{tabular}{|c|c|c|c|c|c|c|}
\hline \multirow[b]{2}{*}{ Gene } & \multicolumn{2}{|c|}{ Basal-like vs. Luminal } & \multicolumn{2}{|c|}{ Basal-like vs. HER2 } & \multicolumn{2}{|c|}{ Luminal vs. HER2 } \\
\hline & Mean difference & P-value & Mean difference & P-value & Mean difference & P-value \\
\hline $\mathrm{CDH} 13$ & -2.611 & 0.533 & -14.022 & 0.006 & -11.411 & 0.012 \\
\hline SFRP1 & -16.089 & $<0.001$ & -14.076 & 0.001 & 2.014 & 0.927 \\
\hline FHIT & -0.047 & 0.879 & -0.541 & 0.137 & -0.494 & 0.121 \\
\hline Syk & 2.376 & 0.198 & 1.392 & 0.510 & -0.984 & 0.571 \\
\hline$R I Z 1$ & -6.313 & 0.113 & -1.744 & 0.704 & 4.570 & 0.236 \\
\hline
\end{tabular}

HER2, human epidermal growth factor receptor 2; CDH13, cadherin 13 (H-cadherin); SFRP1, secreted frizzled-related protein 1; RIZ1, retinoblastoma protein-interacting zinc-finger gene 1 .

Table VI. Association between clinicopathological characteristics and DNA methylation levels (P-value).

\begin{tabular}{lrrrrr}
\hline & CDH13 & SFRPI & FHIT & Syk & RIZ1 \\
\hline Age & 0.950 & 0.823 & 0.512 & 0.345 & 0.042 \\
Menopausal status & 0.859 & 0.633 & 0.316 & 0.889 & 0.015 \\
Stage & 0.519 & 0.299 & 0.203 & 0.848 & 0.797 \\
Tumor size & 0.374 & 0.227 & 0.781 & 0.842 & 0.256 \\
LN(+) & 0.545 & 0.173 & 0.476 & 0.294 & 0.855 \\
Metastasis & 0.307 & 0.180 & 0.187 & 0.284 & 0.237 \\
Histological grade & 0.158 & 0.168 & 0.758 & 0.016 & 0.807 \\
LVI & 0.608 & 0.355 & 0.788 & 0.896 & 0.983 \\
ER & 0.248 & 0.002 & 0.768 & 0.170 & 0.174 \\
PR & 0.138 & 0.015 & 0.428 & 0.176 & 0.380 \\
HER2 & 0.006 & $<0.001$ & 0.498 & 0.819 & 0.637 \\
Ki-67 & 0.194 & 0.162 & 0.759 & 0.027 & 0.125 \\
Bcl-2 & 0.308 & 0.001 & 0.545 & 0.550 & 0.933 \\
p53 & 0.271 & 0.347 & 0.802 & 0.761 & 0.945 \\
EGFR & 0.973 & $<0.001$ & 0.858 & 0.974 & 0.209 \\
Necrosis & 0.534 & 0.064 & 0.715 & 0.676 & 0.751 \\
\hline
\end{tabular}

CDH13, cadherin 13 (H-cadherin); SFRP1, secreted frizzled-related protein 1; $R I Z 1$, retinoblastoma protein-interacting zinc-finger gene 1; LN(+), lymph node status; LVI, lymphovascular invasion; ER, estrogen receptor; PR, progesterone receptor; HER2, HER2, human epidermal growth factor receptor 2; EGFR, epidermal growth factor receptor.
Unsupervised hierarchical clustering based on the 5 genes and the variable methylated $\mathrm{CpG}$ loci of each gene resulted in the formation of multiple small clusters that had no similar biological patterns. As shown in Fig. 2, the results showed classes that were not well separated and that did not appear to be associated with a molecular subtype.

Methylation status and clinicopathological parameters. A comparison of the methylation status with the clinicopathologic characteristics revealed that the negative expression of ER, PR and HER-2, and the positive expression of Bcl-2 and EGFR were associated with a low level of SFRPI gene methylation $(\mathrm{P}=0.002, \mathrm{P}=0.015, \mathrm{P}<0.001, \mathrm{P}=0.001$ and $\mathrm{P}<0.001$, respectively) (Table VI). The correlations between the methylation status of RIZl, Syk and FHIT and the clinicopathological characteristics were dissimilar, RIZI was associated with menopausal status and age $(\mathrm{P}=0.015$ and $\mathrm{P}=0.042$, respectively). Syk was associated with histological grade $(\mathrm{P}=0.016)$.

\section{Discussion}

Gene expression microarray analysis has made it possible to identify distinct molecular subtypes that are associated with different clinical outcomes (1-3). Previous studies have documented the aberrant methylation of $\mathrm{CpG}$ islands in gene promoters in breast carcinogenesis $(7,8)$, and have indicated 


\section{Methylation level}
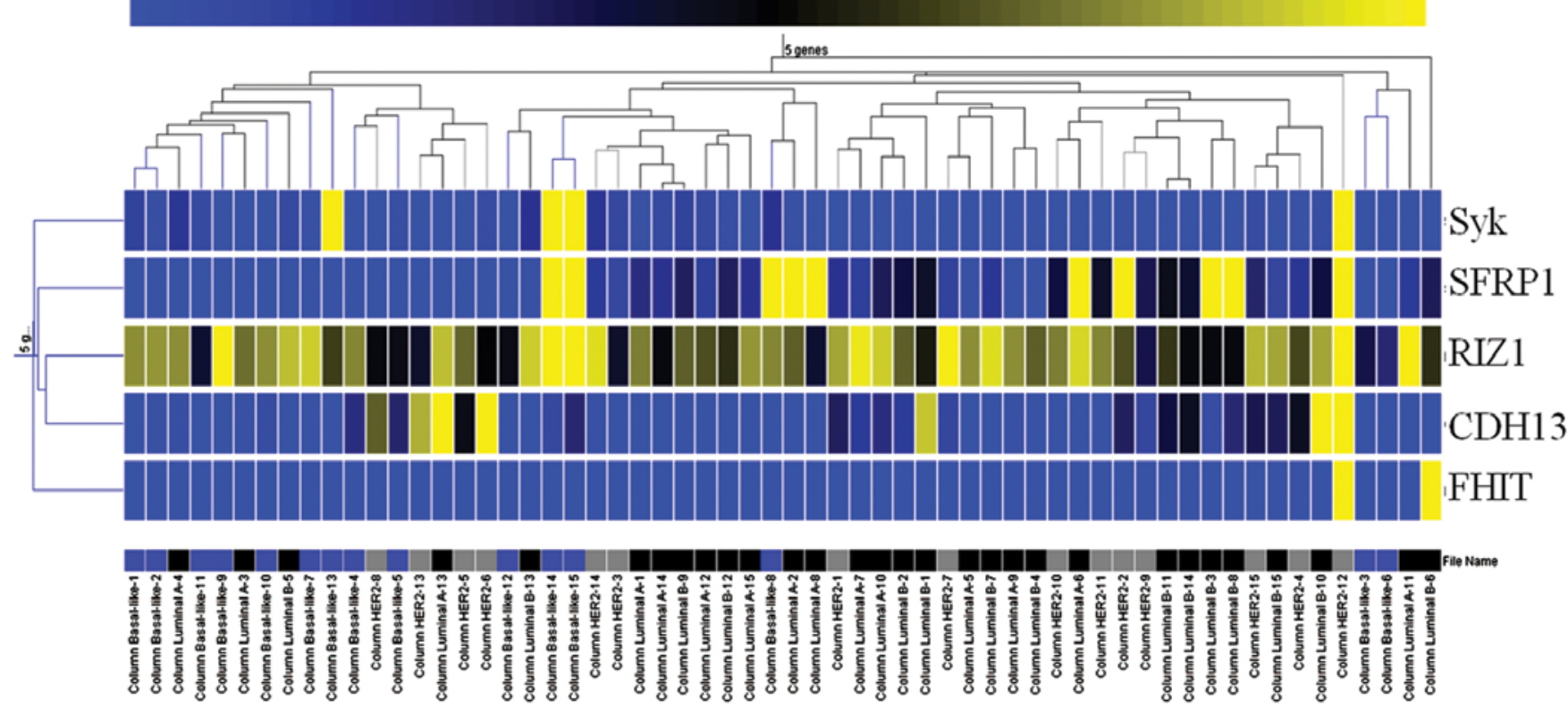

Figure 2. Unsupervised hierarchical clustering of the 5 candidate genes and samples using methylation data. Differential methylation patterns are indicated in yellow vs. blue. As noted, no differentiated clusters were generated by this statistical analysis. CDH13, cadherin 13 (H-cadherin); SFRP1, secreted frizzledrelated protein $1 ; R I Z 1$, retinoblastoma protein-interacting zinc-finger gene 1 .

that specific DNA methylation patterns may be associated with some of the known breast cancer subtypes $(9,10)$.

We observed a significantly lower methylation level at a $\mathrm{CpG}$ site in the SFRPI gene in the basal-like breast cancer subtype when compared with other molecular subtypes, consistent with the results of a previous study (13). SFRPI is a member of the SFRP family (14) and a putative inhibitor of Wnt signaling $(15,16)$ that plays a key role in embryonic development, cell differentiation and proliferation, as well as tumor development and progression (17). It has been described that SFRPI expression is frequently lost or downregulated in breast cancer $(18,19)$, and the loss of SFRP1 expression is associated with poor prognosis, indicating a putative tumor suppressor gene function of SFRPI (19). Furthermore, a recent study indicated that SFRPI has potential as a novel biomarker for the triple-negative breast cancer phenotype (20). Thus, SFRPl hypermethylation may contribute to breast carcinogenesis, and may be useful as a novel prognostic marker of breast cancer, particularly basal-like tumors. In our study, $83.3 \%$ of the breast cancer cases had an aberrant methylation of the SFRPl gene. This result suggests that promoter hypermethylation is the predominant mechanism of SFRPl gene silencing in human breast cancer, as shown in a previous study (21), although $S F R P 1$ protein expression analysis was not included in this study. Of the 60 cases that we analyzed, 15 were of the basal-like subtype and tended to have decreased methylation of the SFRPl gene, suggesting that the DNA methylation of this gene may contribute to the phenotype of breast cancer subtypes, particularly the basal-like subtype.

The basal-like subtype of breast cancer has a triple-negative phenotype with a poor prognosis and no specific targeted therapy, despite an increased response to chemotherapy compared to other breast cancer subtypes. Of note, a recent study reported that, in triple-negative breast cancer, SFRPI expression significantly correlates with an increased sensitivity to neoadjuvant chemotherapy with taxane/anthracycline, and preliminary experiments with siRNA-mediated knockdown of SFRP1 showed a significantly decreased sensitivity to paclitaxel, doxorubicin and cisplatin (20). In our study, the low frequency of SFRP1 methylation in the basal-like subtype may account for the higher expression of SFRPI in basal-like tumors compared to the other breast cancer subtypes and the associated increase in response to chemotherapy in triple-negative breast cancer compared to the other subtypes. These results suggest that SFRP1 signaling and methylation have potential as biomarkers tailored for the basal-like subtype, and may be used to predict chemotherapy response, as well as for treatment selection.

In addition, analysis of genes related with subtype-specific methylation revealed that $C D H 13$ was specifically hypermethylated in HER2 tumors when compared with the other molecular subtypes. This result is in accordance with a previous study stating that the $\mathrm{CDH} 13$ gene is highly methylated in HER2/neupositive breast cancers (22). The $C D H 13$ gene, coding for $\mathrm{H}$-cadherin, is a member of the cadherin family and a putative mediator of cell-cell interaction and cancer cell invasion and metastasis. It is considered as a tumor suppressor gene and may contribute to the enhancement of tumor progression and invasion (23). However, we did not observe a correlation between CDH13 methylation and stage, tumor grade, lymph node status and metastasis, which may reflect the limited sample size used in this study.

Several genes have previously been shown to be aberrantly methylated in breast cancer $(8,24,25)$. Furthermore, breast cancer subtype-specific epigenotypes have been investigated through candidate gene approaches, as well as genome-wide 
DNA methylation analysis. However, these observations require further confirmation as the methylation frequency of a candidate gene and subtype-specific methylation patterns vary between independent studies. For example, Bediaga et al (9) and Holm et al (10) who used the array-based DNA methylation-profiling approach, found that each molecular subtype of breast cancer displayed specific methylation profiles $(9,10)$. However, these studies did not identify and validate specific genes, such as $S F R P 1$ and $C D H 13$, whose methylation status was used to discriminate between the basal-like and HER2 subtypes in our study. Nevertheless, our findings are consistent with those of previous studies, stating that the HER2 subtype is associated with the preferential hypermethylation of several genes, and that basal-like tumors have several genes with low methylation levels $(9,10,22,26)$. Taken together, our results support the findings that methylation may play a significant role in different breast tumor phenotypes.

In the present study, we used quantitative DNA methylation analysis to measure the methylation frequency in 5 genes known to be involved in breast carcinogenesis and are commonly methylated in breast cancer. Pyrosequencing is uniquely capable of quantifying methylation in explicit sequence context, thereby enabling several consecutive $\mathrm{CpG}$ sites to be quantified individually in a single assay. Although approaches for genome-wide DNA methylation analysis hold promise for identifying novel epigenetic targets, pyrosequencing is effective for identifying and quantifying the aberrant methylation of breast cancer genes and determining the criteria that may characterize and discriminate specific molecular subtypes.

To detect possible common patterns of methylation associated with the breast cancer molecular subtypes, hierarchical clustering of the 5 genes was performed. However, the discriminating ability of clustering analysis was poor compared with using the methylation status of the SFRPI gene alone. This may be due to the relatively small sample size and the small number of candidate genes, which were insufficient to produce meaningful results (27). Furthermore, 3 of the 5 candidate genes, FHIT, Syk and RIZl, displayed dissimilar methylation frequencies, and the methylation status of these genes may not be useful for the molecular classification of breast cancer.

Our study had several limitations. First, our study included a relatively small number of samples and candidate genes. These factors limit the application of our results to clinical settings. Further studies with a larger number of breast cancer cases are required to provide additional evidence. In addition, an analysis of additional candidate genes may help define subtype-specific methylation profiling for breast cancer classification. Second, we did not include normal breast tissue in the present study. Studies have shown that candidate genes are hypermethylated in breast tumors, whereas matched normal breast tissues have very low or no methylation levels $(21,22,28-30)$. However, direct comparisons of methylation levels in tumor tissue with those of normal tissue would be required to prove that epigenetic mechanisms may play a key role in the development of breast cancer. Furthermore, comparisons of gene expression with methylation levels in tumor and normal tissue would be helpful to address this issue further.

In conclusion, our study revealed that the basal-like subtype of breast cancer displays distinct methylation profiles compared to other subtypes. We found that the basal-like subtype is associated with low methylation levels of the SFRPI gene, and that the hypermethylation of $\mathrm{CDH} 13$ differs among the molecular subtypes of breast cancer. These results suggest that the analysis of molecular markers, such as gene hypermethylation are useful for the improved characterization of molecular subtypes, and that the methylation levels of specific genes in breast cancer may potentially serve as epigenetic biomarkers and prognostic factors. Further studies with larger sample sizes and more comprehensive DNA methylation profiling with validation are required to clarify the predictive and prognostic value of gene methylation patterns in breast cancer.

\section{Acknowledgements}

The present research has been supported by Korea Breast Cancer Foundation.

\section{References}

1. Sørlie T, Perou CM, Tibshirani R, et al: Gene expression patterns of breast carcinomas distinguish tumor subclasses with clinical implications. Proc Natl Acad Sci USA 98: 10869-10874, 2001.

2. Sørlie T, Tibshirani R, Parker J, et al: Repeated observation of breast tumor subtypes in independent gene expression data sets. Proc Natl Acad Sci USA 100: 8418-8423, 2003.

3. Parker JS, Mullins M, Cheang MC, et al: Supervised risk predictor of breast cancer based on intrinsic subtypes. J Clin Oncol 27: 1160-1167, 2009.

4. Nielsen TO, Hsu FD, Jensen K, et al: Immunohistochemical and clinical characterization of the basal-like subtype of invasive breast carcinoma. Clin Cancer Res 10: 5367-5374, 2004.

5. Cheang MCU, Chia SK, Voduc D, et al: Ki67 index, HER2 status, and prognosis of patients with luminal B breast cancer. $\mathrm{J}$ Natl Cancer Inst 101: 736-750, 2009.

6. Jaenisch R and Bird A: Epigenetic regulation of gene expression: how the genome integrates intrinsic and environmental signals. Nat Genet 33: 245-254, 2003.

7. Dworkin AM, Huang TH and Toland AE: Epigenetic alterations in breast: Implications for breast cancer detection, prognosis and treatment. Semin Cancer Biol 19: 165-171, 2009.

8. Widschwendter M and Jones PA: DNA methylation and breast carcinogenesis. Oncogene 21: 5462-5482, 2002.

9. Bediaga N, Acha-Sagredo A, Guerra I, et al: DNA methylation epigenotypes in breast cancer molecular subtypes. Breast Cancer Res 12: R77, 2010.

10. Holm K, Hegardt C, Staaf J, et al: Molecular sybtypes of breast cancer are associated with characteristic DNA methylation patterns. Breast Cancer Res 12: R36, 2010.

11. Goldhirsch A, Wood WC, Coates AS, et al: Strategies for subtypes - dealing with the diversity of breast cancer: highlights of the St. Gallen International Expert Consensus on the Primary Therapy of Early Breast Cancer 2011. Ann Oncol 22: 1736-1747, 2011.

12. Huang DW, Sherman BT and Lempicki RA: Systematic and integrative analysis of large gene lists using DAVID Bioinformatics Resources. Nat Protoc 4: 44-57, 2009.

13. Wang S, Dorsey TH, Terunuma A, Kittles RA, Ambs S and Kwabi-Addo B: Relationship between tumor DNA methylation status and patient characteristics in African-American and European-American women with breast cancer. PLoS One 7: e37927, 2012.

14. Rattner A, Hsieh JC, Smallwood PM, et al: A family of secreted proteins contains homology to the cysteine-rich ligand-binding domain of frizzled receptors. Proc Natl Acad Sci USA 94: 2859-2863, 1997.

15. Finch PW, He X, Kelley MJ, et al: Purification and molecular cloning of a secreted, Frizzled-related antagonist of Wnt action. Proc Natl Acad Sci USA 94: 6770-6775, 1997.

16. Cadigan KM and Nusse R: Wnt signaling: a common theme in animal development. Genes Dev 11: 3286-3305, 1997.

17. Polakis P: Wnt signaling and cancer. Genes Dev 14: 1837-1851, 2000. 
18. Ugolini F, Adélaïde J, Charafe-Jauffret E, et al: Differential expression assay of chromosome arm $8 p$ genes identifies Frizzledrelated (FRP1/FRZB) and Fibroblast Growth Factor Receptor 1 (FGFR1) as candidate breast cancer genes. Oncogene 18: 1903-1910, 1999.

19. Klopocki E, Kristiansen G, Wild PJ, et al: Loss of SFRP1 is associated with breast cancer progression and poor prognosis in early stage tumors. Int J Oncol 25: 641-649, 2004.

20. Liedtke C, Ruckert C, Goette M, et al: Secreted frizzled receptor protein 1 (sFRP-1) as both a potential novel biomarker of triple negative breast cancer (TNBC), and its sensitivity against taxane/anthracycline containing neoadjuvant chemotherapy. Cancer Res 69 (24 Suppl): 4047, 2009.

21. Veeck J, Niederacher D, An H, Klopocki E, et al: Aberrant methylation of the Wnt antagonist SFRP1 in breast cancer is associated with unfavourable prognosis. Oncogene 25: 3479-3488, 2006.

22. Fiegl H, Millinger S, Goebel G, et al: Breast cancer DNA methylation profiles in cancer cells and tumor stroma: association with HER-2/neu status in primary breast cancer. Cancer Res 66: 29-33, 2006.

23. Celebiler CA, Kilic Y, Saydam S, et al: Predicting invasive phenotype with CDH1, CDH13, CD44, and TIMP3 gene expression in primary breast cancer. Cancer Sci 100 : 2341-2345, 2009.
24. Hinshelwood RA and Clark SJ: Breast cancer epigenetics: normal human mammary epithelial cells as a model system. J Mol Med 86: 1315-1328, 2008.

25. Lo PK and Sukumar S: Epigenomics and breast cancer. Pharmacogenomics 9: 1879-1902, 2008.

26. Feng W, Shen L, Wen S, et al: Correlation between CpG methylation profiles and hormone receptor status in breast cancers. Breast Cancer Res 9: R57, 2007.

27. Eisen MB, Spellman PT, Brown PO and Botstein D: Cluster analysis and display of genome-wide expression patterns. Proc Natl Acad Sci USA 95: 14863-14868, 1998.

28. Yuan Y, Mendez R, Sahin A and Dai JL: Hypermethylation leads to silencing of the SYK gene in human breast cancer. Cancer Res 61: 5558-5561, 2001.

29. Raish M,Dhillon VS, Ahmad A, et al: Promoter hypermethylation in tumor suppressing genes p16 and FHIT and their relationship with estrogen receptor and progesterone receptor status in breast cancer patients from Northern India. Transl Oncol 2: 264-270, 2009.

30. Du Y, Carling T, Fang W, Piao Z, Sheu JC and Huang S: Hypermethylation in human cancers of the RIZ1 tumor suppressor gene, a member of a histone/protein methyltransferase superfamily. Cancer Res 61: 8094-8099, 2001. 